ORIGINAL ARTICLE

\title{
Neonatal transfers by advanced neonatal nurse practitioners and paediatric registrars
}

\author{
A Leslie, T Stephenson
}

Arch Dis Child Fetal Neonatal Ed 2003;88:F509-F512

See end of article for authors' affiliations

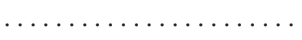

Correspondence to: A Leslie, Neonatal Unit, City Hospital, Hucknall' Road, Nottingham NG5 IPB, UK; aleslie@ ncht.trent.nhs.uk

Accepted

3 December 2002

\begin{abstract}
Objective: To evaluate the safety and practicality of using advanced neonatal nurse practitioners (ANNPs) to lead acute neonatal transfers.

Design: Comparison of transport times, transport interventions, and physiological variables, covering the first four complete years of operating a transport service that uses ANNPs and specialist paediatric registrars (SpRs) interchangeably.

Setting: Tertiary neonatal transport service.

Patients: The first 51 transfers of sick infants under 28 days of age by an ANNP led transport team into Nottingham compared with the next consecutive SpR led transfer after each ANNP led one.

Main outcome measures: Transport times; interventions and support given during stabilisation for transfer and during transfer; condition on completion of transfer, assessed from blood glucose, systolic blood pressure, $\mathrm{pH}$, oxygenation, and temperature.

Results: The ANNP led team responded more rapidly to requests for transfer and took longer to stabilise babies. The groups undertook similar numbers of procedures during stabilisation, and there were no differences in the ventilatory and other support that infants needed in transit. The infants transferred by the doctor led group had worse values for $\mathrm{pH}$ (doctor led, 7.31 (6.50-7.46); ANNP led, 7.35 (7.04-7.50), $\mathrm{p}=0.02$ ) and $\mathrm{PaO}_{2}$ (doctor led, 6.7 (2.4-13.1); ANNP led, 8.7 (3.5-17.0); $\mathrm{p}=0.008$ ) before transfer (all values median (range)). Comparisons of the infant's condition before and after transfer showed a significant improvement in temperature for the infants transferred by ANNP led teams $\left(36.8^{\circ} \mathrm{C}(34.0-\right.$ $\left.37.8) \vee 37.0^{\circ} \mathrm{C}(34.6-38.0), p=0.001\right)$ and in oxygen saturation $(96 \%(88-100) \vee 98 \%(92-100)$, $p=0.01$ ). There were no differences between the ANNP and doctor led groups in the values obtained for any variable after transfer.

Conclusions: Clinical condition on completion of transport is similar for babies transferred by ANNP and doctor led teams. ANNP led transport appears to be practical and safe.
\end{abstract}

$\mathrm{E}$ valuations of the work performed by advanced neonatal nurse practitioners (ANNPs) in the United Kingdom have so far reported on attendance at deliveries ${ }^{1}$ and routine baby checks, ${ }^{2}$ but none has reported the use of ANNPs in acute neonatal transport.

Studies of strategies for improving transport outcomes have concentrated on the staff who attend transfers. Several studies have examined the impact of training the staff who attend transfers to ensure that they have specific transport skills. In the United States, these studies have reported better transport outcomes, such as improvements in temperature and acid base balance after transfer, when a trained team is used for transport. These teams have been led by doctors ${ }^{3}{ }^{4}$ and by nurses. ${ }^{56}$ In the United Kingdom, structured evaluation of staffing arrangements for transport has in the last 10 years consisted only of our earlier audit study, which suggested that sending a trained transport nurse on every transfer as expert support for the registrar was associated with improved transport outcomes. ${ }^{7}$

The established model in the United Kingdom for transport team composition for acute neonatal transport is at least one doctor and one nurse. The doctor is usually a specialist paediatric registrar (SpR), although senior house officers and consultants are also used, as local circumstances dictate. ANNPs may potentially be equipped with the range of skills and knowledge necessary to stabilise and transfer sick newborn infants.

This paper reports the first four complete years of operating a transport service that uses ANNPs and SpRs interchangeably. ANNP led transport became a practical possibility locally in September 1997.

\section{METHODS}

Data were collected contemporaneously on all acute transfers conducted by the Nottingham Neonatal Service (including general details of the baby, the infant's problems, referral centre, timing of each stage of the transfer, equipment used during transfer, equipment problems, maximum ventilation requirements during the transfer, number and type of infusions, and any medical problems with the baby during transfer). The timing data were used to derive a response time (time from acceptance of the referral to departure from the base unit), a stabilising time (arrival at referring unit to departure from that unit), and a return journey time (departure from referring unit to arrival at base unit) for each transfer. Data were also collected on blood glucose, systolic blood pressure, blood $\mathrm{pH}$, oxygenation, and core body temperature at two points in time on each transfer. Data were collected as soon as possible after the team arrived at the referring centre, to reflect the condition of the infant before any stabilisation. Data were also collected after the infant had been transferred from the transport incubator to a static incubator in Nottingham, to reflect the condition of the

Abbreviations: ANNP, advanced neonatal nurse practitioner; SpR, specialist paediatric registrar 
Table 1 Characteristics of the two groups of babies

\begin{tabular}{llll}
\hline & $\begin{array}{l}\text { ANNP led } \\
(\mathbf{n}=51)\end{array}$ & $\begin{array}{l}\text { Doctor led } \\
(\mathbf{n}=51)\end{array}$ & p Value \\
\hline $\begin{array}{l}\text { Gestation at birth (weeks) } \\
\begin{array}{l}\text { Gestation at transfer } \\
\text { (weeks) }\end{array}\end{array} 33(23-41)$ & $32(23-40)$ & 0.9 \\
Weight at transfer (kg) & $1.66(0.53-5.1)$ & $33(23-43)$ & 0.83 \\
Age at transfer (days) & $1(0.2-28)$ & $0.75(0.56-3.91)$ & 0.89 \\
\hline
\end{tabular}

Gestations are completed weeks. Values are median (range). There are no significant differences $(p<0.05)$ between the groups for any category (Mann-Whitney U test).

ANNP, Advanced neonatal nurse practitioner

infant after transfer. ${ }^{7}$ Oxygenation was assessed by arterial sampling $\left(\mathrm{PaO}_{2}\right)$ where possible. As no additional tests were performed beyond those clinically indicated, an oxygen saturation value $\left(\mathrm{SaO}_{2}\right)$ was recorded if no $\mathrm{PaO}_{2}$ was available. Core temperature was measured in the axilla using a Suretemp electronic thermometer.

A transport nurse attends every transfer. Acute transfers are attended by a second team member, either an $\mathrm{SpR}$ or an ANNP. Allocation to ANNP or SpR led transfer is determined by availability. ANNPs are mostly scheduled to work 08001800 on the neonatal intensive care unit, but are extra to the medical and nursing complement at that time, in order that they may attend transfers if required. Out of these hours, and when an ANNP is unavailable for any reason, transport expertise is provided by SpRs. Transfers are not triaged. ANNPs attend any neonatal transfer request that occurs when they are available. The same equipment is used on all transfers.

The SpRs $(\mathrm{n}=34)$ were all paediatric trainees. A transport training day is organised for this group twice a year.

The ANNPs $(n=3)$ each followed ANNP training with a period of consolidation of 9-12 months on their base unit before starting transport training. Transport training comprised attending transfers (15-20) with experienced team leaders, SpRs or ANNPs. The clinical director of the unit conducted a final check off exercise, based around demonstrating clinical problem solving skills and competence at "prescribing".

Data were analysed on all 51 retrievals of neonates less than 28 days of age at the time of transfer by ANNP led teams into a Nottingham neonatal unit between September 1997 and September 2001. These were compared with 51 SpR led transfers conducted over the same period, selected by taking the next consecutive $\mathrm{SpR}$ led retrieval into Nottingham after each ANNP led retrieval.

\section{Statistical methods}

For basic data, medians and ranges are given. Mann-Whitney $\mathrm{U}$ tests and Wilcoxon's signed rank tests were used for comparisons between and within the cohorts. A result was deemed to be significant when $\mathrm{p} \leqslant 0.05$.

\section{RESULTS}

Details of infants transferred

Table 1 gives the characteristics of the infants transferred. Referrals came from a maximum distance of 80 miles. Infants were transferred from a total of 17 units. There are no significant differences between the groups for gestation at birth, gestation at transfer, weight at transfer, or age at transfer. The groups were similar with respect to diagnosis at the time of transfer, the most common diagnosis in both groups being prematurity and respiratory distress syndrome (ANNP, 23/51; SpR, 25/51). The remaining infants had a large number of other diagnoses (ANNP, $\mathrm{n}=18$; $\mathrm{SpR}, \mathrm{n}=16$ ), with small numbers of babies presenting with each.

\section{Times}

Table 2 shows time data. It was not possible to derive a response time for 10 ANNP led transfers or for seven doctor led transfers. This was usually because the team were already committed to another transfer. The ANNP led teams had a significantly faster response time and spent significantly longer in the stabilising period. All the ANNP led transfers started on Monday to Friday between 0800 and 1800. The SpR transfers were also predominantly Monday to Friday activity ( $\mathrm{n}=44$ ), but 32 started after 1800 and before 0800 .

The response time is derived from the time at which the request for transfer was accepted to the time at which the team departed from the base unit. The stabilising time is derived from the time of arrival on the referring unit until the time at which the team departed, the return journey time is the time from departing the referring unit to arrival in Nottingham, and the total transfer time is time from departing base unit to arriving back at base unit. Differences between the groups were assessed using the Mann-Whitney U test.

\section{Procedures}

A tick list was completed contemporaneously by the transport nurse, to indicate procedures both attempted and successfully completed by the ANNP or doctor; details are given in table 3. No distinction was made in the data collection between attempting a procedure, such as umbilical arterial catheter placement, and successful completion. In all infants who required first intubation or reintubation by the transport team, the procedure was successfully completed. There were no differences between the groups (Mann-Whitney U Test) for any procedure. There were non-significant trends for the ANNP led teams to perform more first intubations (where an infant had never been intubated previously) and more umbilical arterial catheters, and for the doctor led teams to perform more reintubations.

\section{Ventilation and other support given in transit}

Table 4 gives details of ventilatory and other support. Of the infants transferred by the ANNP led teams, 34/51 (66\%) were ventilated through an endotracheal tube during the journey. A further $13(25 \%)$ were in supplemental oxygen, but not

\begin{tabular}{llllllll} 
Table 2 & Times involved in transfers & & & \\
\hline & \multicolumn{2}{l}{ ANNP led } & & \multicolumn{2}{l}{ Doctor led } & \\
\cline { 2 - 3 } & No & Median (range) & & No & Median (range) & p Value \\
\hline Response time (min) & 41 & $55(15-285)$ & & 44 & $79(20-345)$ & 0.0007 \\
Stabilising time (min) & 50 & $120(50-280)$ & & 50 & $97.5(30-290)$ & 0.049 \\
Return journey time (min) & 51 & $50(15-110)$ & & 51 & $45(15-110)$ & 0.32 \\
Total time (h) & 51 & $4.75(1.5-8.5)$ & & 50 & $4.77(1.75-8)$ & 0.41 \\
\hline
\end{tabular}

ANNP, Advanced neonatal nurse practitioner. 
Table 3 Procedures attempted or completed by the transport team

\begin{tabular}{lll}
\hline & ANNP led & Doctor led \\
\hline Peripheral arterial catheter & $5(9.8)$ & $5(9.8)$ \\
Umbilical arterial catheter & $5(9.8)$ & $2(3.9)$ \\
Umbilical venous catheter & $1(1.9)$ & $1(1.9)$ \\
First intubation & $6(11.7)$ & $1(1.9)$ \\
Reintubation & $6(11.7)$ & $11(21.5)$ \\
Blood gases & $2(0-4)$ & $1(0-5)$
\end{tabular}

Values are expressed as the number of transfers (percentage of transfers) on which each procedure was attempted, except number of blood gases which shows the number of these performed on each transfer with the range in parentheses. There are no significant differences $(p<0.05)$ between the groups for any category (Mann-Whitney $U$ test). ANNP, Advanced neonatal nurse practitioner.

ventilated. This was not significantly different from the doctor led group: 30/51 (59\%) ventilated, 10 (19\%) in supplemental oxygen. A number of babies in each group were neither ventilated nor receiving supplemental oxygen (ANNP led, 4/51; doctor led, 11/51). These were infants referred with a variety of gastrointestinal surgical diagnoses. There were no significant differences between the groups in the level of ventilatory support needed or supplemental oxygen, whether ventilated or not. All the ventilated infants transferred by ANNP led teams received intravenous opiate sedation during the journey, usually morphine or diamorphine, and 28/30 ventilated infants transferred by the doctor led teams were similarly appropriately sedated.

There were no records of any transport related adverse events during any journey. These were defined as (any of) extubation, intubation, treatment for pneumothorax, or administration of resuscitation drugs.

\section{Clinical condition}

Table 5 shows details of the five physiological variables that could be recorded. These assess whether infants were
Table 4 Support required in transit

\begin{tabular}{lll}
\hline & ANNP led & Doctor led \\
\hline Number ventilated (\%) & $34(66.6)$ & $30(58.8)$ \\
Maximum PIP $\left(\mathrm{cm} \mathrm{H} \mathrm{H}_{2} \mathrm{O}\right)$ & $19(13-35)$ & $20(14-32)$ \\
Maximum PEEP $\left(\mathrm{cm} \mathrm{H} \mathrm{H}_{2} \mathrm{O}\right)$ & $4(2-5)$ & $4(2-6)$ \\
Maximum ventilator rate & $55(6-90)$ & $60(15-76)$ \\
Maximum $\mathrm{FiO}_{2}$ (ventilated infants) & $0.38(0.21-1)$ & $0.39(0.21-1)$ \\
Maximum $\mathrm{FiO}_{2}$ (unventilated infants) & $0.27(0.21-0.6)$ & $0.27(0.21-0.6)$ \\
Ventilated babies receiving sedation & $34(100)$ & $28(93)$ \\
Number of infusions per infant & $2(1-5)$ & $2(1-5)$ \\
Inotropic support & $7(13.7)$ & $3(5.8)$ \\
Intra-arterial BP measurement & $17(33.3)$ & $21(41.1)$ \\
Transcutaneous blood gas monitoring $11(21.5)$ & $5(9.8)$ \\
\hline
\end{tabular}

Data are either median (range) or number (\%). Data on maximum respiratory support refer to infants on ventilation. Data on infusion numbers, inotrope use, blood pressure monitoring and transcutaneous gas monitoring refer to percentage of all transfers. There are no significant differences between the groups (Mann-Whitney $U$ test). ANNP, Advanced neonatal nurse practitioner; PIP, peak inspiratory pressure; PEEP, positive end expiratory pressure; BP, blood pressure.

significantly different before or after transfer in the ANNP led group compared with the doctor led group and compare values obtained before and after transfer for ANNP and doctor led transfers, to assess the effect of stabilisation and transfer by each group on each variable.

The values for $\mathrm{pH}$ and $\mathrm{PaO}_{2}$ obtained before transfer by the doctor led group were significantly worse than those for the ANNP led transfers. Comparisons of infant condition before and after transfer show significant improvements in temperature and $\mathrm{SaO}_{2}$ values for the ANNP led transfers. There are no significant differences in the values obtained for any variable after transfer when the ANNP led transfers are compared with the doctor led ones.

\section{DISCUSSION}

An ANNP led neonatal transport programme has not been previously evaluated in the United Kingdom. These data suggest that when acute neonatal transfers are led by ANNPs,

Table 5 Details of the five physiological variables recorded before and after transfer

\begin{tabular}{|c|c|c|c|}
\hline & Before & After & p Value \\
\hline \multicolumn{4}{|c|}{ Blood glucose $(\mathrm{mmol} / \mathrm{l})$} \\
\hline ANNP led & $4(1.8-9.8)(n=49)$ & $4.2(1-8.6)(n=51)$ & 0.8 \\
\hline Doctor led & $4.3(1.1-15.9)(n=50)$ & $4.6(2.2-11.9)(n=47)$ & 0.11 \\
\hline $\mathrm{p}$ Value & 0.92 & 0.31 & \\
\hline \multicolumn{4}{|c|}{ Systolic blood pressure } \\
\hline ANNP led & $59(27-97)(n=41)$ & $50(31-112)(n=41)$ & 0.29 \\
\hline Doctor led & $56(29-118)(n=38)$ & $56(26-90)(n=36)$ & 0.42 \\
\hline $\mathrm{p}$ Value & 0.44 & 0.85 & \\
\hline \multicolumn{4}{|l|}{$\mathrm{pH}$} \\
\hline ANNP led & $7.35(7.04-7.5)(n=46)$ & $7.35(6.82-7.49)(n=42)$ & 0.83 \\
\hline Doctor led & $7.31(6.5-7.46)(n=39)$ & $7.38(6.55-7.6)(n=36)$ & 0.1 \\
\hline $\mathrm{p}$ Value & 0.02 & 0.97 & \\
\hline \multicolumn{4}{|c|}{ Temperature $\left({ }^{\circ} \mathrm{C}\right)$} \\
\hline ANNP led & $36.8(34-37.8)(n=49)$ & $37(34.6-38)(n=51)$ & 0.001 \\
\hline Doctor led & $36.8(35-37.9)(n=50)$ & $36.8(35.4-37.8)(n=50)$ & 0.24 \\
\hline $\mathrm{p}$ Value & 0.25 & 0.21 & \\
\hline \multicolumn{4}{|c|}{ Oxygenation: $\mathrm{PaO}_{2}(\mathrm{kPa})$} \\
\hline ANNP led & $8.7(3.5-17)(n=21)$ & $8.2(6.6-26)(n=20)$ & 0.92 \\
\hline Doctor led & $6.7(2.4-13.1)(n=22)$ & $8.5(1.89-17.5)(n=21)$ & 0.19 \\
\hline $\mathrm{p}$ Value & 0.008 & 0.9 & \\
\hline \multicolumn{4}{|c|}{ Oxygenation: $\mathrm{SaO}_{2}$ (\%) } \\
\hline ANNP led & $96(88-100)(n=30)$ & $98(92-100)(n=31)$ & 0.01 \\
\hline Doctor led & $96(88-100)(n=29)$ & $97(92-100)(n=30)$ & 0.09 \\
\hline $\mathrm{p}$ Value & 0.85 & 0.08 & \\
\hline
\end{tabular}

Data are median (range). Data are shown for ANNP and doctor led transfers and for the time points before and after transport. In each case, the number of data points available are given. Comparisons between the ANNP and doctor led groups are made using the Mann-Whitney $U$ test, and comparisons of values before and after transport within each group are made using the Wilcoxon signed rank test.

ANNP, Advanced neonatal nurse practitioner. 
the condition of babies on completion of transport is similar to when transfers are led by SpRs.

In our service, the ANNP led teams were able to respond significantly more rapidly to transport requests. Clearly this is not a quality intrinsic to ANNP led teams, but is a result of the supernumerary staff being based on the neonatal unit and immediately available for transport call outs during "office hours". East Midlands Ambulance Service has staggered shift change times, and therefore the differences in response times cannot be explained by the doctor led group consistently requesting vehicles at ambulance shift changeover times. The response time does not include the time spent in transit to the referring unit. This is principally dependent on distance, over which we have no influence.

The stabilising times were significantly different between the groups, with ANNPs taking longer. There is no evidence that the ANNPs are carrying out significantly more procedures, and the data available do not allow other explanations to be investigated. The similarity in the return journey times between the groups suggests that any differences seen in physiological variables are not simply a consequence of longer transit times for one group.

The ventilatory and other support given by each kind of team to infants in transit appears similar, and there were no serious adverse events in transit. This suggests that the groups were both broadly appropriately supported for transfer.

The significant differences in $\mathrm{PaO}_{2}$ and $\mathrm{pH}$ between the groups before transfer suggest that the infants transferred by SpR led teams were more unwell when the team first assessed them than those transferred by the ANNP led group. This raises problems in the comparability of the groups, which may not be disentangled without a randomised study. We can speculate that the infants in the former group may genuinely have been sicker, as reflected in their need to be transferred out of hours and cared for on the referring unit at a time of day when most units have fewer staff. However, it may also be that the worse $\mathrm{PaO}_{2}$ and $\mathrm{pH}$ values were consequent on a feature of the SpR led team, such as the longer response time. We cannot distinguish between these possibilities from our data.

Comparisons of the physiological data obtained before and after transfer suggest that both teams were maintaining or improving the condition of the infant over the stabilisation and transfer periods, and this is congruent with our earlier findings. ${ }^{8}$ Although the infants in the SpR led transfers had worse acid base status before transfer, the groups were similar after. The ANNP led group had improved temperature values after transfer, and, although this is similar to other ANNP assessment projects, ${ }^{1}$ the magnitude of the difference seen may be of limited clinical significance.

The babies studied included a number in both groups who were neither ventilated nor in oxygen. These reflect the workload of a transport team that retrieves infants for neonatal surgery. Although a number of these babies may not need support of airway or breathing, the presence of a doctor or ANNP is often necessary so that other clinical problems, such as fluid and electrolyte status and pain relief, are attended to. Moreover, an infant not requiring respiratory support at the time of referral can deteriorate abruptly and require their specialist skills, especially during a long transport.

Many obstacles had to be overcome for the service to become operational. Facilitating a prescribing like activity for the ANNPs required substantial work, with senior pharmacist support. Patient group directives were produced, in line with the guidance issued by the Department of Health. ${ }^{10}$ ANNPs are at present a more scarce resource than SpRs, and the training issues are substantial. To take an experienced neonatal nurse through ANNP training, subsequent consolidation, and transport training takes about two years. Significant administrative problems also had to be considered: the trust board were asked to approve nurses assuming such a substantial role, and to accept the liability for the actions of the transport team, wherever it was working and however it was constituted. An ANNP led transport team requires commitment from many members of the team at the base hospital and the support of all the clinicians in the service.

In this study we have shown that ANNP led neonatal transport is a practical possibility and appears to be safe for babies. It is not clear whether ANNPs will be cost effective in this role, bearing in mind their lengthy training and supernumerary status. The next evaluation should be a randomised trial, including economic analysis, and subsequent adopters of the ANNP model should consider such studies.

\section{CONCLUSION}

The future configuration of neonatal services in the United Kingdom is currently under review. These data suggest that transport is an area in which ANNPs may contribute to service provision.

\section{Authors' affiliations}

A Leslie, Nottingham Neonatal Service, City and University Hospitals, Nottingham, UK

T Stephenson, Division of Child Health, University Hospital, Nottingham

\section{REFERENCES}

1 Aubrey W, Yoxall C. Evaluation of the role of the neonatal nurse practitioner in resuscitation of preterm infants at birth. Arch Dis Child Fetal Neonatal Ed 2001;85:F96-9.

2 Lee T, Skelton R, Skene C. Routine neonatal examination: effectiveness of trainee paediatricians compared with advanced neonatal nurse practitioners. Arch Dis Child Fetal Neonatal Ed 2001;85:F100-4.

3 Chance G, Matthew J, Gash J, et al. Neonatal transport: a controlled study of skilled assistance. J Pediatr 1978;93:662-6.

4 Hood J, Cross A, Hulka B, et al. Effectiveness of the neonatal transport team. Crit Care Med 1983;11:419-23.

5 Cook L, Kattwinkel J. A prospective study of nurse supervised versus physician supervised neonatal transports. J Obstet Gynecol Neonatal Nurs 1983; 12:371-6.

6 Thompson T. Neonatal transport nurses: an analysis of their role in the transport of newborn infants. Paediatrics 1980;65:887-92.

7 Leslie A, Stephenson T. Audit of neonatal intensive care transport: closing the loop. Acta Paediatr 1997;86:1253-6.

8 Leslie A, Stephenson T. Audit of neonatal intensive care transport. Arch Dis Child Fetal Neonatal Ed 1994;71:F61-6.

9 Crown DJC. Review of prescribing, supply and administration of medicines: a report on the supply and administration of medicines under group protocols. London: Department of Health, 1998.

10 Stephenson T. Implications of the Crown Report and nurse prescribing. Arch Dis Child 2000;83:199-202. 